\title{
EDITORIAL NOTE On the Colombo, Hutto, Myin exchange
}

\author{
Shaun Gallagher
}

Published online: 5 May 2014

(C) Springer Science+Business Media Dordrecht 2014

The following response by Hutto and Myin to Colombo's original paper, "Explaining social norm compliance. A plea for neural representations," contains some inaccurate quotations attributed to Colombo. These inaccuracies are not the fault of the authors, but are due to editorial mistakes and to problems involved in publishing electronic versions online first. Hutto and Myin worked from and were quoting from an earlier draft of Colombo's paper (dated 2012), which is different from the revised version that was first published online in January 2013. Because the inaccuracies were discovered only after Hutto and Myin's response was published online, corrections were not possible. Also, the fact that page numbers are assigned only when hard copy publication occurs, and do not appear in the Online First publication made it impossible for Hutto and Myin, or for Colombo in his response, to provide pagination for quotations. The editors take responsibility and apologize to the authors for failing to check the accuracy of the quotations.

The following is a list of the discrepancies between the published statements in Colombo (2013) and the quoted material in Hutto and Myin (H\&M).

- H\&M: "Colombo (2012) tells us that in order for a footballer to comply, on the fly, with the relevant social norms the individual must be 'sensitive to abstract, relational properties such as the values 'fair play', 'reciprocity' or 'cheating"',

Colombo (2013) states: "One condition for the player to comply with this norm is that he is sensitive to abstract, relational properties such as "fair play," "reciprocity," and "cheating"".

- H\&M: "the special relationship between neural activations and the sources with which such activations correlate 'seems to constitute a code' (Colombo 2012)."

Colombo (2013): "Neural activations and the sources, or variables, with which they correlate, can be said to constitute a code, viz. a mapping from some source alphabet to some target alphabet."

- H\&M: "The code in question is said to specify the relevant "functional relationships' (Colombo 2012, p. 9). This leads him to conclude that we are licensed to 'say that neural representations are the constituents of the neural code' (Colombo 2012, p. 9)."

S. Gallagher $(\square)$

Department of Philosophy, The University of Memphis, Memphis, USA

e-mail: s.gallagher@memphis.edu 
Colombo (2013): "The neural code specifies functional relationships between properties of neural activity and properties of internal or external variables... neural representations can be said the constituents of the neural code."

- H\&M: "We are told that, "Neural encoding refers to the transduction of some stimulus', whereas, 'Neural decoding refers to the extraction of information about some stimulus from neural spiking' (Colombo 2012, p. 9)."

Colombo (2013): "Neural encoding refers to the transduction of some external stimulus (or internal variable) by the system resulting in the spiking of one or more neurons....

Neural decoding refers to the extraction of information about some external stimulus (or internal variable) from neural spiking."

- H\&M: "Even more vague is Colombo's account of the way decoding 'determines how the visual information carried by the activity in a certain neural population yields perceptual visual belief' (Colombo 2013, p. 10)."

Colombo (2013): "Decoding determines how the visual information carried by activity in a certain neural population yields a perceptual visual belief and is used by the rest of the system to generate behaviour (Colombo 2010 provide further details about this notion of neural representing and its relationship with intentionality)."

- H\&M: "Finally, Colombo also tells us that "to understand the scope, motivations, aims and current practice in cognitive science, invoking neural representations is often necessary"”.

Colombo (2013): "to understand current explanatory practice in cognitive science, invoking neural representations is often necessary." 